Neurosurg Focus 9 (4):Intro, 2000

\title{
Introduction to Thoracic Disc Disease
}

\author{
Charles B. Stillerman, M.D.
}

In the October issue of Neurosurgical Focus we examine thoracic disc disease, which remains an interesting and challenging problem. Symptomatic disc herniations are uncommon in the thoracic spine. Surgery for herniated thoracic discs comprises fewer than $5 \%$ of all disc operations. Historically, treatment of this disorder has been problematic because of delays in diagnosis resulting from both the infrequency of this disease and its lack of a characteristic presentation pattern. Moreover, the indications for surgery have not been well established because the natural history of this disorder is not clearly defined. An absolute indication for surgery is generally regarded to be the presence of severe and/or progressive myelopathy. The role of surgery to control pain is more uncertain. Many patient-care issues relate to the pathophysiology of this disease. McInerney and Ball begin this edition with a comprehensive discussion of this field.

Selection of the optimum surgical approach for disc removal is also controversial. Initially, laminectomy was used to treat thoracic disc disease; however, the approach was plagued by suboptimum outcomes. As a result of this early experience, new approaches to the thoracic spine evolved, providing improved access to the ventral spinal canal and disc space. Recognized leaders in this field have discussed several of these approaches. The surgical indications and techniques section begins with Kalfas' interesting discussion of a rarely encountered lesion, thoracic stenosis, which is perhaps the one clear indication for laminectomy in the treatment of thoracic disc disease. Presently there are several posterolateral approaches used in the removal of thoracic discs. The first of these, described in 1978 by Patterson and Arbit, is the transpedicular approach. Bilsky provides an excellent review of this technique as well as results of a clinical series. Jho describes his modification of the technique using endoscopic assis- tance. Transfacet pedicle-sparing and laminotomy/mesial facetectomy procedures are also presented. The lateral costotransversectomy approach is described by Kim, et al., who combine conventional costotransversectomy techniques with computer-assisted image guidance as an adjunctive tool for preoperative planning and navigation.

Vollmer and Simmons discuss the anterolateral transthoracic approach to the thoracic spine. They provide an insightful presentation of preoperative considerations, relevant anatomy, and surgical techniques. Thoracoscopic discectomy is a new and evolving treatment option. Burke and Caputy provide a historical perspective on the surgical treatment of thoracic disc herniations, emphasizing the thoracoscopic approach. Das and Rothberg detail the history of thoracoscopy in a clear and concise fashion. Johnson and colleagues offer a comprehensive examination of the indications and techniques of thoracoscopic discectomy, presenting a surgical series and discussing the results and complications. Chen provides a thorough analysis of several large contemporary thoracic disc series, whereas McCormick, et al., offer an in-depth presentation of complications and their avoidance. In closing Bao and Yuan discuss the technology of artificial disc replacement and speculate on its future role in the treatment of thoracic disc disease.

I would like to thank the following individuals for their thoughtful manuscript reviews: Martin H. Weiss, M.D., Edward C. Benzel, M.D., Regis W. Haid, Jr., M.D., Iain H. Kalfas, M.D., Thomas C. Chen, M.D., Marc E. Eichler, M.D., Eric J. Woodard, M.D., and Leslie A. Sebring, M.D. I also express my gratitude to Ms. Susan Robinson, Production Editor of Neurosurgical Focus, for her commitment to making this edition achieve our expectations. Finally, an additional and special thanks to Dr. Martin H. Weiss for his unwavering guidance and support. 